\title{
Studies on the Antibiotic Substances from Actinomyces. 15th Report.
}

\author{
(Preliminary note) \\ By \\ Masahiko Kuroya, Nakao Ishida, Ken Katagiri,

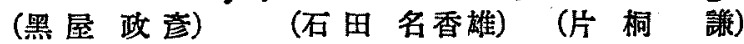 \\ Bunji Takahashi and Takehiko Shiratori. \\ (高橋 交治) (白取 剛䆣) \\ (From the Department of Bacteriology, Faculty of Medicine, \\ Tohoku University, Sendai.) \\ (Received for publication August 27, 1951) \\ 1. Eight strains of Actinomyces belonging to streptothricin type ( 1 and \\ 2) and the purified active $\mathrm{HCl}$ salts isolated from them were examined com- \\ paratively upon their " antibacterial spectra " using several kinds of test organ- \\ isms including streptomycin resistant $E$. coli and 4 strains of $E$. coli which were \\ made resistant to each of 4 different kinds of streptothricin respectively. All \\ streptothricin resistant $E$. coli strains were resistant to each of $\mathrm{HCl}$ salt solutions \\ of both types of streptothricins in a similar way, so it was not possible in this way \\ to differentiate both types of streptothricins from each other. They were useful, \\ however, in order to differentiate the culture of Streptomyces themselves, be- \\ cause the streptothricin resistant $E$. coli strains were somewhat differently \\ subceptible according to their own types, even if they were far more resistant \\ 2) A strain of Streptomyces (O-1) showed a streptothricin-like spectrum \\ in a state of culture on the plate, while the other strain (488) in a state of culture \\ filtrate and finally the third strain (149) in a state of pure $\mathrm{HCl}$ salt solution, \\ showed a similar spectrum. This fact indicated that there were many incom- \\ formities between the spectra produced by organisms, by their culture filtrates \\ or by the solutions of isolated antibiotics, and may be explained by the assump- \\ tion, that the antibiotics produced by Streptomyces culture on the plates may \\ exceed both, qualitiatively and quantitatively that contained in their culture \\ filtrates and may contain other antibiotics different from that yielded in a state
} than the original strains of $E$. coli. of $\mathrm{HCl}$ salt solutions. 